\title{
A Novel Geometrical Mixed-Bounce Two-Ring Deterministic Model for MIMO Mobile-to-Mobile Non- Isotropic Communication Channel
}

\author{
MALA Umar Mustapha \\ Bakura \\ University of Maiduguri, Nigeria
}

\author{
Bala Alhaji Salihu \\ Beijing University of Posts and \\ Telecommunications China
}

\author{
Suleiman Zubair \\ UTM MIMOS CoE Universiti \\ Teknologi Malaysia Johor, \\ Malaysia
}

\begin{abstract}
An accurate modeling of multiple-input multiple-output (MIMO) channels is a prerequisite for designing multiantenna system. In this paper, we propose a geometrical mixed-bounce two-ring deterministic model for mobile-tomobile channel considering the non-isotropic scattering while adopting the Von Mises probability density function for both the angle of departure AoD and angle of arrival AoA surrounding the transmitter and receiver respectively. Beginning with isotropic scattering, the expression for two dimensional (2-D) space time cross-correlation (STCC) function between any two sub channels is derived. Comparison between the statistical properties of the deterministic model under the assumption of non-isotropic scattering with that of the reference model indicates a good agreement, thus, the importance of this model. The result obtained is in conformity with that of the double bounce and single bounce two ring models.
\end{abstract}

\section{General Terms}

Model.

\section{Keywords}

Mixed bounce, mobile-to-mobile channel, space time correlation, Isotropic scattering.

\section{INTRODUCTION}

Designing a very high speed minimum error wireless link having good quality of service (QoS) and with considerable range capabilities in a highly fading channel attracts significant research interest and poses a great challenge in communication engineering. Assuming no fading for the moment, we can, in principle, meet the $1-\mathrm{Gb} / \mathrm{s}$ data rate requirement if the product of bandwidth $(\mathrm{Hz})$ and spectral efficiency $(\mathrm{b} / \mathrm{s} / \mathrm{Hz})$ equals $10^{9}$. As clearly contained in literature, a variety of cost, technology, and regulatory constraints make such a brute force solution unattractive, if not impossible. In an effort to achieve high data rate wireless communication services with less interference and fading, communication engineers have recently concentrated much of their resources and time on the new dimension- the space. It is now a well-established fact that higher data rates are achievable by employing MIMO which entails the application of array of antennas both at the transmitter and receiver utilizing the space-time technology. The analysis and simulation of space-time wireless communication systems need accurate, yet tractable spatio-temporal channel models. Here we aim at providing a novel mixed-bounce mobile-tomobile deterministic channel model assuming non-isotropic scattering of electromagnet waves. Non isotropic scattering is a good approximation as it is more near to real phenomena. It is natural that the multipath signal components received at any antenna may consist of the single-bounce, the double-bounce and even both of them. Single-bounce literally means signals from the transmitting antenna undergo a single hop through a scatterer before arriving at the receiving antenna while double bounce involves two hops before arriving at the receiver. We in this paper are incorporating all the signal paths in determining the channel transfer function and further looking at some of its statistical properties. In literature many geometrically based MIMO channel models were proposed such as the one ring, two ring, multi-ring and spherical models. In the two ring model both single bounce [1] and [2] and double bounce [3] were discussed. To the best of our knowledge no work was done in mixed-bounce where the link between the antenna-scatterer-antenna and antenna-scattererscatterer-antenna are considered together which constitutes the central point of concentration of this paper.

The rest part of the paper is arranged as follows. Section two discusses the two-ring model; section three gives the crosscorrelation; Section four for computation of AoD and AoA while section five is for simulation and result and conclusion is in section six..

\section{THE GEOMETRY OF THE TWO- RING MIXED-BOUNCE MODEL}

The geometry of the two-ring mixed-bounce is shown in fig.1 for a MIMO mobile-to-mobile channel. For simplicity two transmit antennas and two receive antennas are considered where local scatterer of $s_{T}^{k}$ and $s_{R}^{l}$ are equally distributed on the circumference of a separate rings around the transmitter and receiver respectively. The paramount difference between our model and other two ring models is that in our model both the single and double bounce rays were considered simultaneously, since each multipath contributes to the total system's data rate. As could be easily observed from Fig. 1, the local scatterers around the transmitter represented as $s_{T}^{k}$

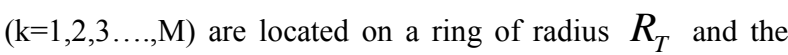
local scatterers around the receiver denoted as $s_{R}^{l}$ $(1=1,2,3 \ldots . . \mathrm{N})$ lie on a separate ring with radius $R_{R}$. The symbols $\phi_{t}^{k}$ and $\psi_{R}^{l}$ represent the main angle of departure 


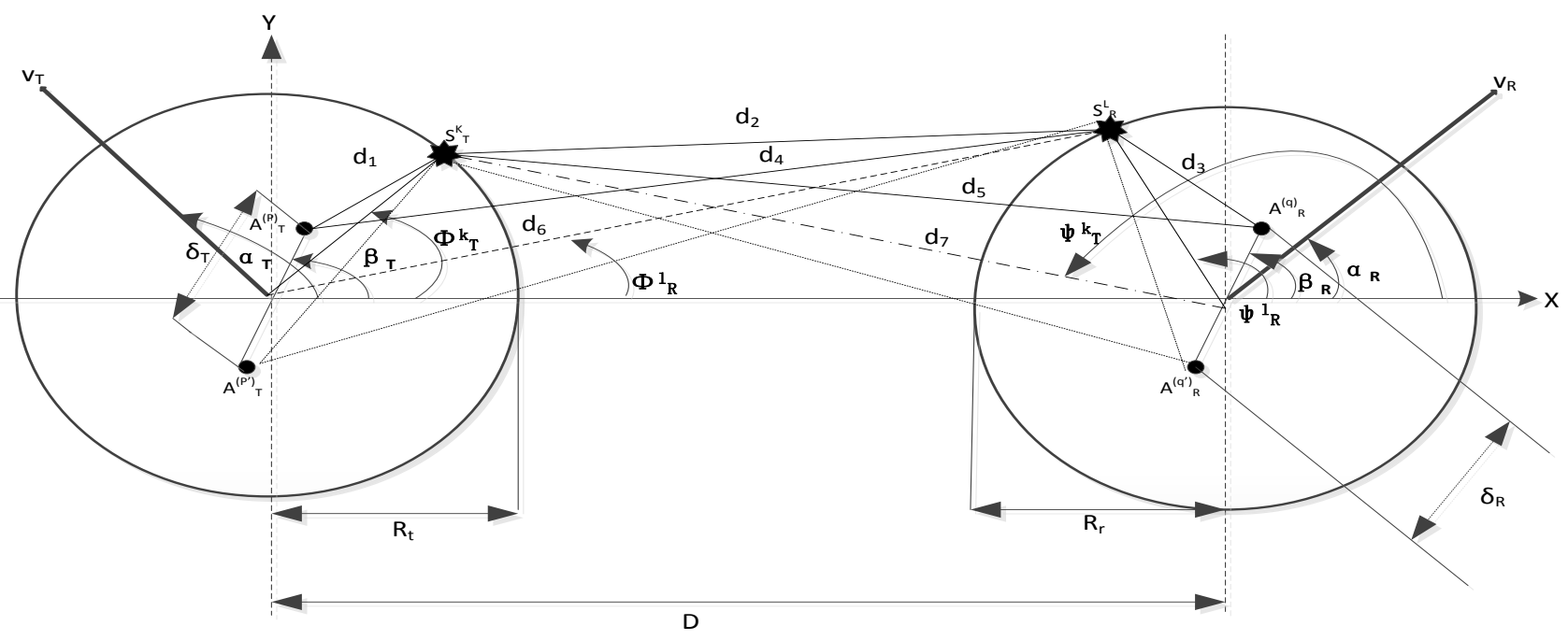

Fig. 1 Mixed Bounce Two Ring Model

(AoD) and main angle of arrival (AoA) respectively and the auxiliary AoD and AoA are represented by $\phi_{R}^{l}$ and $\psi_{T}^{k}$ respectively. It is assumed that the radii $R_{T}$ and $R_{R}$ are small in comparison with $\mathrm{D}$, which is the distance between the transmitter and receiver.

The symbols $\delta_{T}$ and $\delta_{R}$ are used to represent the antenna spacing at the transmitter and receiver respectively. Based on our assumption the inequalities $\max \left\{R_{\mathrm{T}}, R_{R}\right\} \min \left\{\delta_{T}, \delta_{R}\right\}$ and $\min \left\{R_{T}, R_{R}\right\} \square \max \left\{\delta_{T}, \delta_{R}\right\}$ holds. The tilt angle between the $\mathrm{x}$-axis and the orientation of the transmit antenna arrays is denoted by $\beta_{T}$ and the tilt angle of the receiver antenna array is given by $\beta_{R}$.
Both the transmitter and receiver are assumed to move with the velocities of $v_{T}$ and $v_{R}$ respectively in the direction denoted $\alpha_{T}$ and $\alpha_{R}$. Furthermore, $2 \Delta_{T}$ is the maximum angle spread at the transmitter determined by the scatterers around the receiver. Similarly $2 \Delta_{R}$ represents the maximum angle spread at the receiver determined by the scattering around the transmitter. The geometrical two ring model is an appropriate approximation for scenarios in which neither the transmitter nor receiver is elevated but both are surrounded by a large number of scatterers. Such scattering condition may exist both in indoor and outdoor environment. Using cosine rule and other mathematical simplifications, the effective channel transfer function is

$h_{p q}(t)=$

$P_{T S} \lim _{k \rightarrow \infty} \frac{d_{\text {const }}}{\sqrt{M}} \sum_{K=1}^{M} g_{T}^{k} a_{m} \exp \left\{j \frac{\pi \delta_{R}}{\lambda} \cos \left(\beta_{R}-\psi_{T}^{k}\right)+j \Psi_{T}^{k}+j 2 \pi t\left(f_{T_{\max }} \cos \left(\alpha_{T}-\phi_{T}^{k}\right)+f_{R_{\max }} \cos \left(\alpha_{R}-\psi_{T}^{k}\right)\right)\right\}$

$+P_{R S} \lim _{l \rightarrow \infty} \frac{d_{\text {const }}}{\sqrt{N}} \sum_{l=1}^{N} g_{R}^{l} b_{n} \exp \left\{j \frac{\pi \delta_{T}}{\lambda} \cos \left(\beta_{T}-\phi_{R}^{l}\right)+j \Psi_{R}^{l}+j 2 \pi t\left(f_{T_{\max }} \cos \left(\alpha_{T}-\phi_{R}^{l}\right)+f_{R_{\max }} \cos \left(\alpha_{R}-\psi{ }_{R}^{l}\right)\right)\right\}$

$+P_{S_{T R}} \lim _{k, l \rightarrow \infty} \frac{1}{\sqrt{M N}} \sum_{k, l=1}^{M, N} g_{T R}^{k l} g_{m n} \exp \left\{j \theta_{0}+j \Psi_{T R}^{k l}+j 2 \pi \mathrm{t}\left(f_{T}^{(m)}+f_{R}^{(n)}\right)\right\}$

Where

$$
\begin{aligned}
& g_{m n}=a_{m} b_{n} c_{m n} \\
& a_{m}=\exp \left(j \pi\left(\frac{\delta_{T}}{\lambda}\right) \cos \left(\beta_{T}-\phi_{T}^{k}\right)\right. \\
& d_{\text {const }}=\exp \left\{-j \frac{4 R_{T} \pi}{\lambda}\right\}=\exp \left\{-j \frac{4 R_{R} \pi}{\lambda}\right\} \\
& c_{m n}=\exp \left\{j \frac{2 \pi}{\lambda}\left(R_{T} \cos \left(\phi_{T}^{k}-\phi_{R}^{l}\right)-R_{R} \cos \psi_{R}^{l}\right)\right\}
\end{aligned}
$$

$$
\begin{aligned}
& f_{T}^{(m)}=f_{T_{\max }} \cos \left(\alpha_{T}-\phi_{T}^{k}\right) \\
& f_{R}^{(n)}=f_{R_{\max }} \cos \left(\alpha_{R}-\psi_{R}^{l}\right) \\
& \theta_{0}=-\frac{2 \pi}{\lambda}\left(R_{T}+R_{R}+D\right)
\end{aligned}
$$




$$
\begin{aligned}
& R_{p q, p^{\prime} q^{\prime}}\left(\delta_{T}, \delta_{R}, \tau\right)=R_{p q, p^{\prime} q^{\prime}}^{S B T}\left(\delta_{T}, \delta_{R}, \tau\right)+R_{p q, p^{\prime} q^{\prime}}^{S B R}\left(\delta_{T}, \delta_{R}, \tau\right)+R_{p q, p^{\prime} q^{\prime}}^{D B}\left(\delta_{T}, \delta_{R}, \tau\right) \\
& R_{p q, p^{\prime} q^{\prime}}\left(\delta_{T}, \delta_{R}, \tau\right)= \\
& \lim _{k \rightarrow \infty} \frac{P_{T S}}{M} \sum_{K=1}^{M} E\left[\left|g_{T}^{k}\right|^{2}\right] \exp \left\{-j \frac{2 \pi}{\lambda}\left(-\delta_{T} \cos \left(\beta_{T}-\phi_{T}^{k}\right)-\delta_{R} \cos \left(\beta_{R}-\psi_{T}^{k}\right)+j 2 \pi t\left(f_{T_{\max }} \cos \left(\alpha_{T}-\phi_{T}^{k}\right)+f_{R_{\max }} \cos \left(\alpha_{R}-\psi_{T}^{k}\right)\right)\right\}\right. \\
& +\lim _{l \rightarrow \infty} \frac{P_{R S}}{N} \sum_{l=1}^{N} E\left[\left|g_{R}^{l}\right|^{2}\right] \exp \left\{-j \frac{2 \pi}{\lambda}\left(-\delta_{R} \cos \left(\beta_{R}-\psi_{R}^{l}\right)-\delta_{T} \cos \left(\beta_{T}-\phi_{R}^{l}\right)\right)+j 2 \pi t\left(f_{T_{\max }} \cos \left(\alpha_{T}-\phi_{R}^{l}\right)+f_{R_{\max }} \cos \left(\alpha_{R}-\psi_{R}^{l}\right)\right)\right\} \\
& +\lim _{k, l \rightarrow \infty} \frac{P_{S_{T R}}}{M N} \sum_{k, l=1}^{M, N} E\left\{\left[\left|g_{T R}^{k l}\right|^{2}\right] a_{m}^{2} b_{n}^{2} e^{-j 2 \pi t\left(f_{T}^{(m)}+f_{R}^{(n)}\right)}\right. \\
& \cos \left(\beta_{R}-\psi_{T}^{K}\right) \approx-\cos \beta_{R}+\Delta_{T} \sin \beta_{R} \sin \Theta_{T}, \cos \left(\alpha_{R}-\psi_{T}^{K}\right) \approx-\cos \alpha_{R}+\Delta_{T} \sin \alpha_{R} \sin \Theta_{T} \\
& \cos \left(\beta_{T}-\phi_{R}^{l}\right) \approx \cos \beta_{T}+\Delta_{R} \sin \beta_{T} \sin \Theta_{R} \text { and } \cos \left(\alpha_{T}-\phi_{R}^{l}\right) \approx \cos \alpha_{T}+\Delta_{R} \sin \alpha_{T} \sin \Theta_{R} \\
& \int_{-\pi}^{\pi} \exp (x \sin \alpha+y \cos \alpha) d \alpha=2 \pi I_{0}\left(\sqrt{x^{2}+y^{2}}\right) \\
& \text { Let } A_{T}=\exp \left(-j \frac{2 \pi}{\lambda} \delta_{R} \cos \beta_{R}+j 2 \pi t f_{R_{\max }} \cos \alpha_{R}\right), A_{R}=\exp \left(j \frac{2 \pi}{\lambda} \delta_{T} \cos \beta_{T}-j 2 \pi t f_{T_{\max }} \cos \alpha_{T}\right) \\
& B_{\cos _{T}}=j \frac{2 \pi}{\lambda} \delta_{T} \cos \left(\beta_{T}\right)-j 2 \pi t f_{T_{\max }} \cos \alpha_{T}, B_{\cos _{R}}=j \frac{2 \pi}{\lambda} \delta_{R} \cos \left(\beta_{R}\right)+j 2 \pi t f_{R_{\max }} \cos \alpha_{R} \\
& B_{s i n_{R}}=-j 2 \pi t f_{R_{\max }} \sin \alpha_{R}+\mathrm{j} \frac{2 \pi}{\lambda} \delta_{R} \sin \beta_{R}+j \frac{2 \pi}{\lambda} \delta_{T} \Delta_{R} \sin \beta_{T}-j 2 \pi t f_{T_{\max }} \Delta_{R} \sin \alpha_{T} \\
& C_{D_{T}}=j \frac{2 \pi}{\lambda} \delta_{T} \cos \left(\beta_{T}\right)-j 2 \pi t f_{R_{\max }} \cos \alpha_{T} \text { and } C_{D_{R}}=j \frac{2 \pi}{\lambda} \delta_{R} \cos \left(\beta_{R}\right)-j 2 \pi t f_{T_{\max }} \cos \alpha_{R} \\
& D_{D_{T}}=j \frac{2 \pi}{\lambda} \delta_{T} \sin \beta_{T}-j 2 \pi t f_{R_{\max }} \sin \alpha_{T} \text { and } D_{D_{R}}=j \frac{2 \pi}{\lambda} \delta_{R} \sin \beta_{R}-j 2 \pi t f_{T_{\max }} \sin \alpha_{R}
\end{aligned}
$$

As in [1] the diffuse component of $h_{p q}(t)$ follows that the mean value and mean power are equal to 0 and 1respectively hence the central limit theorem state that $h_{p q}(t)$ is a zero-mean complex Gaussian process with unit variance. Consequently the envelope $\left|h_{p q}(t)\right|$ is a Rayleigh fading process. One can show that the diffuse component of the Link from $A_{T}^{p^{\prime}}$ to $A_{R}^{q^{\prime}}$ can be obtained from (1) by replacing by their respective complex conjugates. The rest elements of the matrix could be obtained as in [3].

\subsection{The Space-Time Cross Correlation Function CCF}

The non-normalized space-time correlation function between two complex faded envelopes $h_{p q}$ and $h_{p q}{ }^{\prime}$ is defined

as $R_{p q, p^{\prime} q^{\prime}}\left(\delta_{T}, \delta_{R}, \tau\right)=\left(E\left[h_{q}(t) h_{p_{q^{\prime}}^{\prime}}^{*}(t+\tau)\right]\right)$. From [5] the effective space-time correlation function for the mixed bounce can be calculated using the formula in equation (10) where the superscripts SBT, SBR, and DB stands for Single Bounce Transmitter, Single Bounce Receiver and Double Bounce respectively. The effective STCC for the reference model reduces to the following expression after some mathematical operations and reductions as in equation (11). Furthermore, setting $\sin \varepsilon \approx 0$ and $\cos \varepsilon \approx 1$ we obtain equations (12) and (13).

For values of $\mathrm{M}$ and $\mathrm{N}$ approaching infinity the discrete angle of departure and discrete angle of arrival becomes continuous random variable AOD and continuous random variable AOA where each of which are characterize by certain distribution represented as and respectively. The infinitesimal power contribution due from each scatterer around the transmitter and receiver corresponding to the differential angle and is given as:

$$
\begin{aligned}
& \frac{E\left[\left|g_{T}^{k}\right|^{2}\right]}{M}=p_{\phi_{T}}\left(\phi_{T}\right) d \phi_{T}, \quad \frac{E\left[\left|g_{R}^{l}\right|^{2}\right]}{N}=p_{\psi_{R}}\left(\psi_{R}\right) d \psi_{R} \text { and } \\
& \frac{E\left[\left|g_{T R}^{k l}\right|^{2}\right]}{M N}=p_{\phi_{T}}\left(\phi_{T}\right) p_{\psi_{R}}\left(\psi_{R}\right) d \phi_{T} d \psi_{R} .
\end{aligned}
$$

For non-isotropic scattering, the AoD and AoA have nonuniform distribution over $[-\pi, \pi]$. From [9] we get equation (14) - (19).

The Von Mises also known as circular normal distribution describes a "normal" distribution on a circular path of $2 \pi$. Given by formula in equation (20).

$$
f(\theta \mid \mu, k)=\frac{\exp [k \cos (\theta-\mu)]}{2 \pi I_{0}(k)}
$$

For non- isotropic distribution the Von Mises distribution is considered. For represents isotropic distribution and I0 is the zero order Bessel function and $\square$ is the mean AoA or AoD Various other non-uniform distributions such as Gaussian, quadratic, Laplacian and cosine were discussed in other literatures but due to mathematical suitability the Von Mises 


$$
\begin{aligned}
& R_{p q, p^{\prime} q^{\prime}}\left(\delta_{T}, \delta_{R}, \tau\right) \\
& =\frac{I_{0}\left(\sqrt{k_{R}^{2}+C_{D_{R}}^{2}+D_{D_{R}}^{2}+2 k_{R}\left(C_{D_{R}} \cos \mu_{R}+D_{D_{R}} \sin \mu_{R}\right)}\right) \times I_{0}\left(\sqrt{+k_{T}^{2}+C_{D_{T}}^{2}+D_{D_{T}}^{2}+2 k_{T}\left(C_{D_{T}} \cos \mu_{T}+D_{D_{T}} \sin \mu_{T}\right)}\right) \times P_{S_{T R}}}{I_{0}\left(k_{T}\right) \times I_{0}\left(k_{R}\right)} \\
& +\frac{I_{0}\left(\sqrt{k_{T}^{2}+B_{\cos _{T}}^{2}+B_{\sin _{T}}^{2}+2 k_{T}\left(B_{\cos _{T}} \cos \mu_{T}+B_{\sin } \sin \mu_{T}\right)}\right) P_{T S} A_{T}}{I_{0}\left(k_{T}\right)} \\
& +\frac{I_{0}\left(\sqrt{k_{R}^{2}+B_{\cos _{R}}^{2}+B_{\sin _{R}}^{2}+2 k_{R}\left(B_{\cos _{R}} \cos \mu_{R}+B_{\sin _{R}} \sin \mu_{R}\right)}\right) P_{R S} A_{R}}{I_{0}\left(k_{R}\right)}
\end{aligned}
$$

is adopted in this paper. Substituting equations (11), (12) and (15) to (19) into 11 and undergoing other manipulations we obtain equation (21).

\section{THE SIMULATION MODEL}

It is very difficult if not impossible to implement the reference model either using hardware or software since it involves infinity harmonics, therefore the need to come up with the simulation model. We begin by the stochastic simulation model considering some of its properties such as its spacetime CCF after which we also discuss the deterministic simulation model by applying the principles of deterministic channel modeling along with some of its basic properties as in SSM [11].

\subsection{The Stochastic Simulation Model SSM}

The SSM system can easily be derived from the reference model by considering finite number of scatterers both around the transmitter $\mathrm{M}$ and the receiver $\mathrm{N}$. Of course the scatterers are responsible for the multipath propagation ensuring multiple copies of the transmitted signals at the receiver. Even though we have finite harmonics at the receiver, the phase angles $\theta_{n}, \theta_{m}$ and $\theta_{m n}$ are still i.i.d. random variables each with uniform distribution on the interval $(0,2 \pi]$ thus we have stochastic simulation model SSM where its channel transfer function is given by $\hat{h}_{p q}(t)$. This is for the link between,

The 3-D space -time CCF between two different links $\hat{h}_{p q}(t)$ and $\hat{h}_{p^{\prime} q^{\prime}}(t)$ is calculated using the formula $\hat{\rho}_{p q, p q^{\prime}}\left(\delta_{T}, \delta_{R}, \tau\right)=E\left\{\hat{h}_{p q}(t) \hat{h}_{p^{\prime} q^{\prime}}^{*}(t+\tau)\right\} . \quad$ In the equation above $(.)^{*}$ represent complex conjugate and $E\{$.$\} is$ the expectation operator, which applies to the random phases $\theta_{n}, \theta_{m}$ and $\theta_{m n}$. The closed form expression is therefore

$$
\begin{aligned}
& \hat{\rho}_{p q, p^{\prime} q^{\prime}} \\
& =P_{T S} \exp \left[-j \frac{2 \pi}{\lambda} \delta_{R} \cos \beta_{R}+j 2 \pi f_{R_{\max }} \tau \cos \alpha_{R}\right] \\
& \left.I_{0}\left\{\begin{array}{l}
\left(\begin{array}{l}
-\left(\frac{2 \pi}{\lambda}\right)^{2} \delta_{R} \Delta_{T} \sin \beta_{R}\left(\delta_{R} \Delta_{T} \sin \beta_{R}+2 \delta_{T} \sin \beta_{T}\right) \\
-\frac{(2 \pi)^{2}}{\lambda} \delta_{T}\left(\frac{\delta_{T}}{\lambda}-2 \pi f_{T_{\max }} \tau \cos \left(\alpha_{T}-\beta_{T}\right)\right)-\left(2 \pi f_{T_{\max }} \tau\right)^{2} \\
+\frac{(2 \pi)^{2}}{\lambda} \delta_{R} \Delta_{T} \tau \sin \beta_{R}\left(f_{T_{\max }} \sin \alpha_{T}+\Delta_{T} f_{R_{\max }} \sin \alpha_{R}\right) \\
-(2 \pi)^{2} f_{R_{\max }} \tau \Delta_{T} \sin \alpha_{R}\left(f_{R_{\max }} \tau \Delta_{T} \sin \alpha_{R}-\frac{2}{\lambda} \delta_{T} \sin \beta_{T}+2 f_{T_{\max }} \tau \sin \alpha_{T}\right)
\end{array}\right)
\end{array}\right\}\right)
\end{aligned}
$$

Next, we introduce the information theoretic channel capacity of the SSM. The capacity [4] in the absence of channel knowledge at the transmitter is given by $\hat{C}(t)=\log _{2}\left[\operatorname{det}\left(\boldsymbol{I}_{M_{R}}+\frac{P_{T}}{M_{T} P_{N}} \hat{\boldsymbol{H}}(t) \hat{\boldsymbol{H}}^{H}(t)\right)\right]$ where $\hat{\boldsymbol{H}}(t)=\left[\hat{h}_{p q}(t)\right]$ assuming $M_{T} \geq N_{R}, \boldsymbol{I}_{M_{R}}$ is the $M_{R} \times M_{R}$ identity matrix, $P_{N}$ is the noise power, $P_{T}$ is the total transmitted power allocated uniformly to all $M_{T}$ transmit antenna element. $(.)^{H}$ is the conjugate complex transpose operator. The signal to noise ratio (SNR) is given by $\frac{P_{T}}{P_{N}}$ gives the statistical average of the stochastic capacity with respect to the random phases.

\subsection{The Deterministic Simulation Model DSM}

By making the phase angles and all other parameters of SSM constant, a new model called Deterministic Simulation Model is obtained. Observing rightly that the diffuse component of the link between $A_{T}^{(p)}$ and $A_{R}^{(q)}$ is an ergodic process permits us to consider the properties of the single sample of $\hat{h}_{p q}(t)$ to represent the ensemble properties of the system denoted by $\tilde{h}_{p q}(t)$. If the phases are outcome of a random generator with uniform distribution in the interval $(0,2 \pi]$, $\theta_{n}, \theta_{m}$ and $\theta_{m n}$ are no longer a random variables but constant quantities. Appropriate choice of parameters of $\tilde{h}_{p q}(t)$ leads to very good and close approximation of $h_{p q}(t) . \quad \tilde{h}_{p q}(t)$ is a deterministic function of time therefore statistical properties of DSM is analysed using time averages rather than statistical averages. The 3-D space-time CCF for example is computed thus $\tilde{\rho}_{p q, p^{\prime} q^{\prime}}\left(\delta_{T}, \delta_{R}, \tau\right)=\left\langle\hat{h}_{p q}(t) \hat{h}_{p^{\prime} q^{\prime}}^{*}(t+\tau)\right\rangle$ where $\langle$.$\rangle denotes$ the time average operator. According to [11, Pg. 66], the relation $\tilde{\rho}_{p q, p^{\prime} q^{\prime}}\left(\delta_{T}, \delta_{R}, \tau\right)=\hat{\rho}_{p q, p^{\prime} q^{\prime}}\left(\delta_{T}, \delta_{R}, \tau\right)$ holds .This is applicable to ergodic systems. The DSM's channel capacity is written as

$$
\tilde{C}(t)=\log _{2}\left[\operatorname{det}\left(\boldsymbol{I}_{M_{R}}+\frac{P_{T}}{M_{T} P_{N}} \tilde{\boldsymbol{H}}(t) \tilde{\boldsymbol{H}}^{H}(t)\right)\right]
$$

where $\tilde{\boldsymbol{H}}(t)=\left[\tilde{h}_{p q}(t)\right]$ is deterministic and $M_{T} \geq N_{R}$. The time average $m_{\tilde{C}}$ of channel capacity $\tilde{C}(t)$ is given by the relation $m_{\tilde{C}}=<\tilde{C}(t)>=\lim _{T \rightarrow \infty} \frac{1}{2 T} \int_{-T}^{T} \tilde{C}(t) d t$. The stochastic 
capacity $\hat{C}(t)$ is mean ergodic if $m_{\hat{C}}(t)$ is independent of time $t$ and equal $m_{\tilde{C}}$ thus $m_{\hat{C}}=m_{\tilde{C}}$ [11, Pg. 79].

\section{ASSUMING NON- \\ ISOTROPIC SCATTERING}

4. COMPUTATION of AoD and AoA

On choosing appropriate values for $\mathrm{M}$ and $\mathrm{N}$, in the simulation model we are only left with AoD $\phi_{T}^{k}$ and AoA $\psi_{R}^{l}$ to be determined. For nonisotropic distribution the Modified Method of Equal Areas (MMEA) is applicable in order to calculate the parameters. This method involves finding the set of AoAs or AoDs $\left\{\phi_{T}^{k}\right\}$ such that the ACF for the reference model is approximately equal to ACF of the deterministic model $\quad r_{\mu \mu}(\tau) \approx \tilde{r}_{\mu \mu}(\tau), \quad \tau \in\left[0, \tau_{\max }\right]$ for $N=M \geq 20$. Using numerical root finding techniques [12], the AoD and AoA are determined from the expressions (23) and (24)

$$
\begin{gathered}
\frac{m-1 / 4}{M}-\int_{\phi_{T}^{(0)}-\Pi}^{\phi_{T}^{(m)}} p_{\phi_{T}}\left(\phi_{T}\right) d \phi_{T}=0, m=1,2, \ldots \ldots \ldots . ., M \\
\frac{n-1 / 4}{N}-\int_{\varphi_{T}^{(0)}-\Pi}^{\varphi_{T}^{(m)}} p_{\varphi_{R}}\left(\varphi_{T}\right) d \varphi_{R}=0, n=1,2, \ldots \ldots \ldots \ldots ., N
\end{gathered}
$$

The closed form AoA and AoD are computed if the inverse of the function exist by

$$
\phi_{T}=\psi_{R}=F^{-1}\left(\phi_{T}\right)\left(\frac{n-1 / 4}{N}\right)
$$

The MMEA has the advantages that the statistical properties matches those of the reference model for only small time delay while requiring higher values of scatterers $(\mathrm{M}, \mathrm{N}=40)$ to match the properties of the reference model.

\section{SIMULATION AND RESULTS}

After determining the composite space time cross correlation functions for the mixed bounce two ring model, we hereby present the result of a special case of isotropic scattering using the following model parameters. The antenna tilt angle $\beta_{T}=\beta_{R}=\pi / 2$. The transmit antenna is assumed to move at $\alpha_{T}=\pi / 4$ while the receiver antenna moves at $\alpha_{R}=0$. Identical maximum Doppler frequency is assumed at both the transmitter and receiver given as $91 \mathrm{~Hz}$ and wavelength $\lambda=0.15 \mathrm{~m}$. Under an isotropic scattering we arrive at equations (26) and (27)

Fig. 2 is the mixed bounce composite cross correlation of the entire channel. When we critically observe the figure we find that the it is averaging around zero which clearly indicates

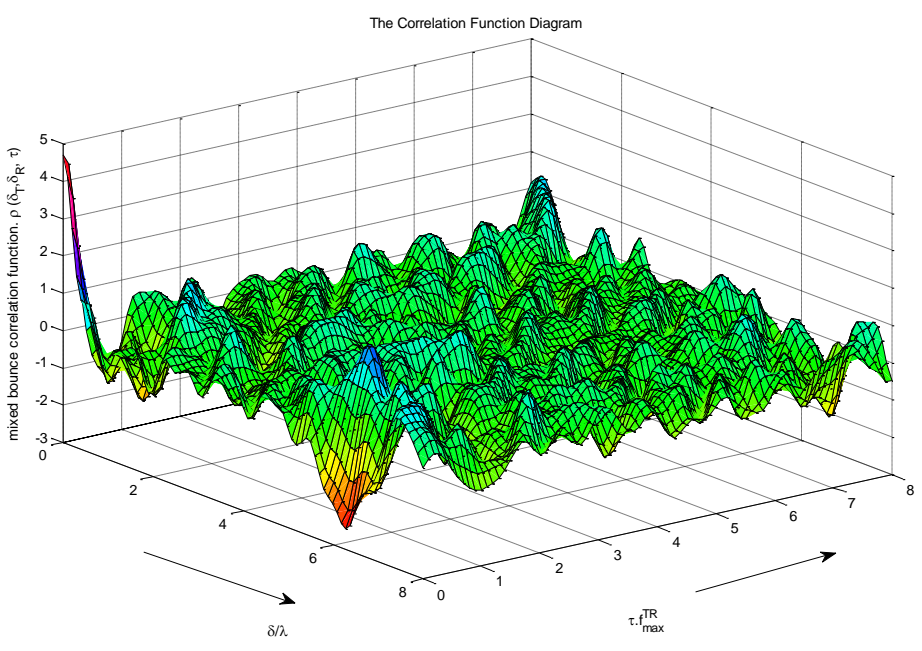

that the correlation is zero and thus it means that the interference between co-channel is less when using MIMO system.

$$
p_{\phi_{T}}\left(\phi_{T}\right) d \phi_{T}=p_{\psi_{R}}\left(\psi_{R}\right) d \psi_{R}=1 /(2 \pi)
$$

Fig 2 Mixed bounce cross correlation function

\section{CONCLUSION}

Here a mixed bounce MIMO mobile-to-mobile deterministic channel model is presented using the two rings as a basis structure. Our simulation model was derived from nonrealizable reference model by applying the concept of deterministic channel modeling. It is assumed that both the transmitter and the receiver antenna are surrounded by finite number of scatterer with a given radius and are also assumed to be moving at a given Doppler frequency. The homogenous channel transfer function is presented while the space-time cross correlation was derived. Our model particularly extended the work done by Matthias Pätzold et al by considering mixed bounce. 


\section{REFERENCES}

[1] huangquan Wang, Raghukumar K., Abdi A., Wallace J., Jensen M., Indoor MIMO channels: a parametric correlation model and experimental results. Symposium on Advances in Wired and Wireless Communication, 2004 IEEE/Sarnoff. Publication Year: 2004 , Page(s): 1 - 5.

[2] Tang Z., Mohan A.S., A correlated indoor MIMO channel model. Canadian Conference on Electrical and Computer Engineering, 2003. IEEE CCECE 2003. Publication Year: 2003 , Page(s): 1889 - 1892 vol.3

[3] Patzöld M.,Hogstad B.O., Youssef N., Dongwoo Kim. A MIMO Mobile-To-Mobile Channel Model: Part I - The Reference Model. IEEE 16th International Symposium on Personal, Indoor and Mobile Radio Communications, 2005. Volume: 1 PIMRC 2005. Page(s): 573 - 578

[4] Hogstad B.O., Patzold M.,YoussefN., Dongwoo Kim. A Mimo Mobile-To-Mobile Channel Model: Part II - The Simulation Model. IEEE 16th International Symposium on Personal, Indoor and Mobile Radio Communications, 2005. PIMRC 2005 Volume: 1 Publication Year: 2005 , Page(s): 562 567

[5] Zajic A.G., Stubber G. Space-Time Correlated Mobile-To-Mobile Channels: Modelling And Simulation. IEEE Transactions on Vehicular Technology, Volume: 57 , Issue: 2. Publication Year: 2008 , Page(s): $715-726$
[6] Xiang Cheng, Cheng-Xiang Wang; Laurenson, D.I.; Hsiao-Hwa Chen; Vasilakos, A.V.; A Generic Geometrical-Based MIMO Mobile-to-Mobile Channel Model. International Wireless Communications and Mobile Computing Conference, 2008. IWCMC '08. Publication Year: 2008 , Page(s): $1000-1005$

[7] Bakhshi, G.; Saadat, R.; Shahtalebi, K.; A modified two-ring reference model for MIMO Mobile-toMobile communication channels. International Symposium on Telecommunications, 2008. IST 2008. Publication Year: 2008 , Page(s): 409 - 413

[8] Gregory D. Durgin "Space-Time Wireless Channel" Prentice Hall PTR, Upper Saddle River, NJ07458.

[9] I.S Gradshteyn and I.M Ryzhik "Table of Integrals, Series, and Product" (Seventh Edition).Academic Press.

[10] David Tse, Pramod Viswanath "Fundamentals of Wireless Communication" Cambridge

[11] M. Patzöld. Mobile Fading Channels, John Willey and Sons. 2002 